\title{
The pulmonary sequalae in discharged patients with COVID-19: a short-term observational study
}

Dehan Liu ${ }^{1,2 \dagger}$, Wanshu Zhang ${ }^{1,2 \dagger}$, Feng Pan ${ }^{1,2}$, Lin Li ${ }^{1,2}$, Lian Yang ${ }^{1,2^{*}}$, Dandan Zheng ${ }^{3}$, Jiazheng Wang ${ }^{3}$ and Bo Liang ${ }^{1,2}$

\begin{abstract}
Background: A cluster of patients with coronavirus disease 2019 (COVID-19) pneumonia were discharged from hospitals in Wuhan, China. We aimed to determine the cumulative percentage of complete radiological resolution at each time point, to explore the relevant affecting factors, and to describe the chest $\mathrm{CT}$ findings at different time points after hospital discharge.
\end{abstract}

Methods: Patients with COVID-19 pneumonia confirmed by RT-PCR who were discharged consecutively from the hospital between 5 February 2020 and 10 March 2020 and who underwent serial chest CT scans on schedule were enrolled. The radiological characteristics of all patients were collected and analysed. The total CT score was the sum of non-GGO involvement determined at discharge. Afterwards, all patients underwent chest CT scans during the 1st, 2nd, and 3rd weeks after discharge. Imaging features and distributions were analysed across different time points.

Results: A total of 149 patients who completed all CT scans were evaluated; there were 67 (45.0\%) men and 82 (55.0\%) women, with a median age of 43 years old (IQR 36-56). The cumulative percentage of complete radiological resolution was $8.1 \%$ (12 patients), $41.6 \%$ (62), 50.3\% (75), and 53.0\% (79) at discharge and during the 1st, 2nd, and 3rd weeks after discharge, respectively. Patients $\leq 44$ years old showed a significantly higher cumulative percentage of complete radiological resolution than patients $>44$ years old at the 3 -week follow-up. The predominant patterns of abnormalities observed at discharge were ground-glass opacity (GGO) (125 [83.9\%]), fibrous stripe (81 [54.4\%]), and thickening of the adjacent pleura (33 [22.1\%]). The positive count of GGO, fibrous stripe and thickening of the adjacent pleura gradually decreased, while GGO and fibrous stripe showed obvious resolution during the first week and the third week after discharge, respectively. "Tinted" sign and bronchovascular bundle distortion as two special features were discovered during the evolution.

Conclusion: Lung lesions in COVID-19 pneumonia patients can be absorbed completely during short-term followup with no sequelae. Two weeks after discharge might be the optimal time point for early radiological estimation.

Keywords: COVID-19, CT, Follow-up, Discharge, Sequalae, GGO, Fibrous stripe

\footnotetext{
* Correspondence: yanglian@hust.edu.cn

${ }^{\dagger}$ Dehan Liu and Wanshu Zhang contributed equally to this work.

'Department of Radiology, Union Hospital, Tongji Medical College,

Huazhong University of Science and Technology, Jiefang Avenue No.1277,

Wuhan 430022, China

${ }^{2}$ Hubei Province Key Laboratory of Molecular Imaging, Wuhan 430022, China

Full list of author information is available at the end of the article
} 


\section{Background}

Coronavirus disease 2019 (COVID-19) has become a worldwide outbreak since the first report in December 2019 in Wuhan, China. By 6 April 2020, 1,210,956 infected patients and 67,594 fatalities had been reported [1], representing a high capability of transmission and a lethal feature. Previous studies have revealed the radiological features at different stages of the disease [2-4], but the radiological manifestations during the convalescence period are still unclear. Wu et al. reported that residual pulmonary lesions such as GGO and intralobular and interlobular septal thickening could be persistently observed years after recovery from severe acute respiratory syndrome (SARS) [5]. It has remained a concern to medical professionals and the public whether similar severe sequalae also exist for COVID-19. This observational study aims to provide the radiographic manifestations of COVID-19 in discharged patients with chest CT follow-up.

\section{Methods}

This study was approved by the Ethics Committees of Union Hospital, Tongji Medical College, Huazhong University of Science and Technology, and it followed the 1964 Helsinki Declaration and its later amendments or comparable ethical standards.

\section{Definition and criteria}

The diagnostic criteria of COVID-19 pneumonia followed the diagnosis and treatment protocols from the National Health Commission of the People's Republic of China. The discharge criteria were as follows: 1 . afebrile for more than 3 days; 2 . respiratory symptoms significantly improved; 3 . improvement in radiological abnormalities on chest radiography or $\mathrm{CT}$; and 4. two consecutive negative COVID-19 nucleic acid tests detected at least $24 \mathrm{~h}$ apart [6]. Complete radiological resolution was defined as the absence of any chest radiographic abnormality potentially related to infection [7].

\section{Patients}

A total of 149 discharged patients previously diagnosed with COVID-19 in this single centre from 5 February 2020 to 10 March 2020 underwent serial chest CT scans on schedule until complete radiological resolution, including 21 patients who were preliminarily reported in the previous study (3). However, in this study, more patients were involved for further exploration, and the same aim was not considered.

\section{Thin-section CT scan and radiological follow-up}

Thin-section CT scanning was performed on a commercial multi-detector CT scanner (Philips Ingenuity
Table 1 Basic characteristics and laboratory examination for patients with COVID-19

\begin{tabular}{|c|c|}
\hline & $\boldsymbol{n}=149$ \\
\hline \multicolumn{2}{|l|}{ Gender } \\
\hline Male & $67(45.0 \%)$ \\
\hline Female & $82(55.0 \%)$ \\
\hline Age $(y)$ & $43(36-56)$ \\
\hline \multicolumn{2}{|l|}{ Triage $^{a}$} \\
\hline Pneumonia & $142(95.3 \%)$ \\
\hline Severe pneumonia & $7(4.7 \%)$ \\
\hline \multicolumn{2}{|l|}{ The initial symptoms of onset } \\
\hline Fever & $121(83.4 \%)$ \\
\hline Cough & 55 (37.9\%) \\
\hline Fatigue & $38(26.2 \%)$ \\
\hline Myalgia & $24(16.6 \%)$ \\
\hline Dyspnea & $15(10.3 \%)$ \\
\hline Diarrhea & $9(6.2 \%)$ \\
\hline \multicolumn{2}{|l|}{ Medical history } \\
\hline Hypertension & $16(11.0 \%)$ \\
\hline Diabetes & $6(4.1 \%)$ \\
\hline Bronchial asthma & $4(2.8 \%)$ \\
\hline Coronary heart disease & $3(2.1 \%)$ \\
\hline \multicolumn{2}{|l|}{ Laboratory examinations at discharge } \\
\hline Lymphocyte count $\left(\times 10^{9}\right.$ cells per L) (1.1-3.2) & $1.54(1.24-1.93)$ \\
\hline D-dimer $(0-0.5 \mu \mathrm{g} / \mathrm{ml})$ & $0.32(0.19-0.56)$ \\
\hline CT score at discharge ${ }^{b}$ & $1(0-2)$ \\
\hline
\end{tabular}

${ }^{\mathrm{a} T h e}$ clinical triage was based on the diagnosis and treatment protocols of pneumonia caused by a novel coronavirus (trial version 7 )

${ }^{\mathrm{b}} \mathrm{A}$ semi-quantitative $\mathrm{CT}$ scoring system was used to estimate the pulmonary involvement of only non-GGO lesions (includes: fibrous stripe and mixed patterns)

Core128, Philips Medical Systems, Best, the Netherlands; SOMATOM Definition AS, Siemens Healthineers, Germany) with a single inspiratory phase. The tube voltage was set as $120 \mathrm{kVp}$ with automatic tube current modulation. From the raw data, CT images were reconstructed with a matrix size of $512 \times 512$ as axial images (thickness of $1.5 \mathrm{~mm}$ and increment of $1.5 \mathrm{~mm}$ ) with hybrid iterative reconstruction (iDose level 5, Philips Medical Systems, the Netherlands) or a

Table 2 The cumulative percentage of complete radiological resolution at different time points

\begin{tabular}{ll}
\hline & $n=149$. \\
\hline Chest CT at discharge & $12(8.1 \%)$ \\
The 1st CT follow-up & $62(41.6 \%)$ \\
The 2nd CT follow-up & $75(50.3 \%)$ \\
The 3rd CT follow-up & $79(53.0 \%)$ \\
\hline
\end{tabular}


pulmonary B70F kernel and a mediastinal B30f kernel (Siemens Healthineers, Germany).

Chest CT scans were regularly performed at different time points, including at discharge and during the 1st, 2nd, and 3rd weeks after discharge. The conventional CT score with residual non-ground-glass opacity was evaluated at the time of CT performed at discharge as for the baseline estimation ( 5 lobes, score 1-5 for each lobe, range, 0 none, 25 maximum). Each of the 5 lung lobes was visually scored from 0 to 5 as: 0 , no involvement; $1,<5 \%$ involvement; 2, 5-25\% involvement; 3, 26-49\% involvement; 4, 50-75\% involvement; 5 , > 75\% involvement. The total CT score was the sum of the individual lobar scores and ranged from 0 (no involvement) to 25 (maximum involvement) [8]. In addition, radiological characteristics at different time points, including GGO, fibrous stripe, thickening of the adjacent pleura, bronchovascular bundle distortion and small pleural effusion, were collected and estimated. The GGO was defined as hazy area of increased attenuation without obscuration of the underlying vasculature [9-11]. If complete radiological resolution was reached, no further chest CT scan was performed.

All image analysis was performed using the institutional digital database system (Vue PACS, version 11.3.5.8902, Carestream Health, Canada) by two radiologists (B.L. and L.Y. who had 25 and 22 years of experience in thoracic radiology, respectively), and final scores were determined by consensus.

\section{Statistical analysis}

Statistical analysis was performed using IBM SPSS Statistics Software (version 24; IBM, New York, USA). Quantitative data are presented as the median with interquartile range (IQR), and the counting data are presented as the percentage of the total unless otherwise specified. The cumulative percentage of the endpoints was calculated. The comparisons of counting data were evaluated using the Chi-square test. A $p$-value of less than 0.05 was defined as statistically significant.

\section{Results}

\section{Basic characteristics}

A total of 149 patients with a male to female ratio of 67 : 82 were included in the study. The average age was 43
Table 3 Correlative factors with resolution

\begin{tabular}{|c|c|c|c|}
\hline & \multicolumn{2}{|c|}{ Complete radiological resolution (n) } & \multirow{2}{*}{$\begin{array}{l}\boldsymbol{p}^{-} \\
\text {value }^{\mathrm{a}}\end{array}$} \\
\hline & Yes & No & \\
\hline \multicolumn{4}{|l|}{ Age (y) } \\
\hline$\leq 44$ & 58 & 26 & $<0.001$ \\
\hline$>44$ & 21 & 44 & \\
\hline \multicolumn{4}{|l|}{ Gender } \\
\hline Male & 39 & 28 & 0.251 \\
\hline Female & 40 & 42 & \\
\hline \multicolumn{4}{|c|}{ CT score at discharge } \\
\hline$\leq 1$ & 60 & 54 & 0.864 \\
\hline$>1$ & 19 & 16 & \\
\hline
\end{tabular}

${ }^{\mathrm{a} C h i-s q u a r e ~ t e s t ~}$

years old (IQR 36-56). The most common initial symptoms were fever $(83.4 \%)$ and cough (37.9\%). Comorbidities of hypertension, diabetes, bronchial asthma, and coronary heart disease were reported in 11.1, 4.1, 2.8, and $2.1 \%$ of the patients, respectively. The median lymphocyte count $\left(1.54 \times 10^{9} / \mathrm{L}\right.$, IQR $1.24-1.93$, normal range: $\left.1.1-3.2 \times 10^{9} / \mathrm{L}\right)$ and D-dimer level $(0.32 \mu \mathrm{g} / \mathrm{ml}$, IQR $0.19-0.56$, normal range: $0-0.5 \mu \mathrm{g} / \mathrm{ml}$ ) were almost normal at discharge. The details are summarized in Table 1.

\section{Cumulative percentage of complete radiological resolution at different time points}

At discharge, 12 (8.1\%) patients reached complete radiological resolution. After that, the cumulative percentage of complete radiological resolution was $41.6 \%$ (62 patients), 50.3\% (75), and 53.0\% (79) during the 1st, 2nd, and 3rd weeks after discharge, respectively (Table 2 ).

\section{Factors associated with resolution}

There was no significant difference in complete radiological resolution at the 3-week follow-up between males and females and between $\leq 1$ and $>1$ CT scores at discharge, while a significant difference was observed between groups with different ages (Table 3 ).

\section{Dynamic chest CT features}

The predominant patterns of abnormalities observed at discharge included GGO (125 [83.9\%]), fibrous stripe (81

Table 4 Chest CT manifestation in the short-term follow-up

\begin{tabular}{lllll}
\hline CT manifestation & CT at discharge & 1st CT follow-up & 2nd CT follow-up & 3rd CT follow-up \\
\hline GGO & $125(83.9 \%)$ & $82(55.0 \%)$ & $69(46.3 \%)$ & $67(45.0 \%)$ \\
Fibrous stripe & $81(54.4 \%)$ & $59(39.6 \%)$ & $50(33.6 \%)$ & $14(9.4 \%)$ \\
Bronchovascular bundle distortion & $10(6.7 \%)$ & $7(4.7 \%)$ & $7(4.7 \%)$ & $6(4.0 \%)$ \\
Thickening of the adjacent pleura & $33(22.1 \%)$ & $27(18.1 \%)$ & $10(6.7 \%)$ & $1(0.7 \%)$ \\
Small pleural effusion & $3(2.0 \%)$ & $2(1.3 \%)$ & $0(0.0 \%)$ & $0(0.0 \%)$ \\
\hline
\end{tabular}




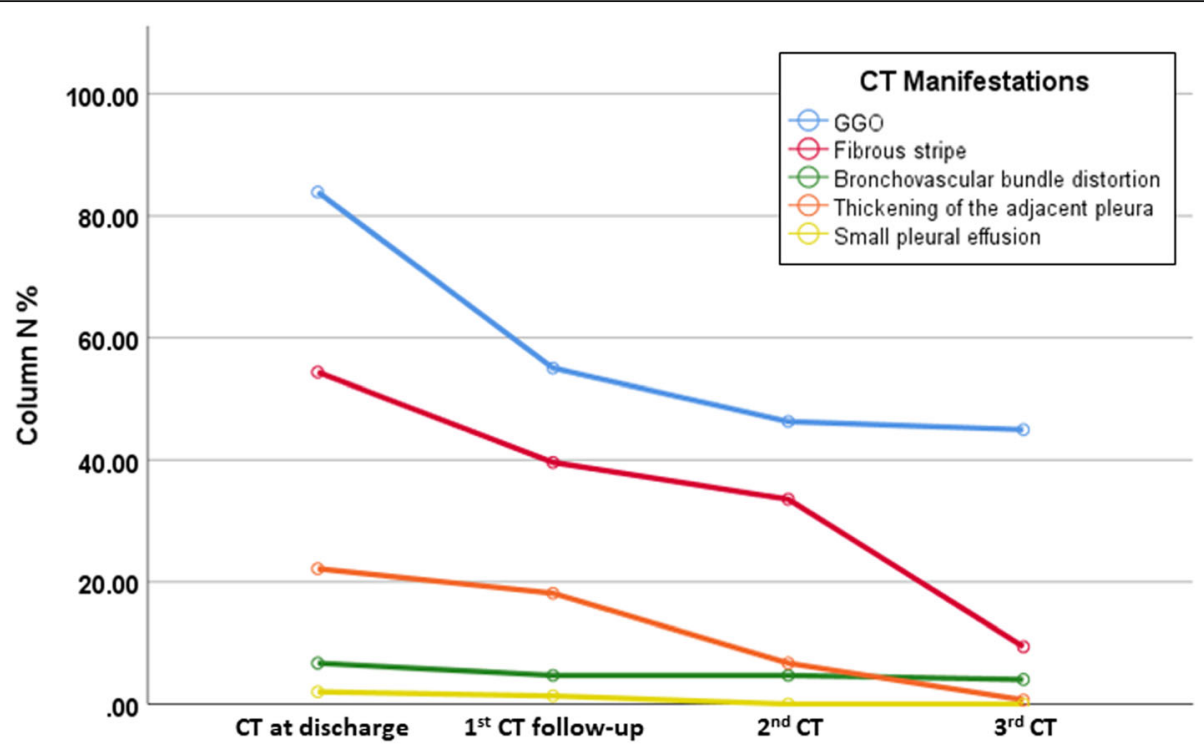

Fig. 1 Dynamic changes of chest $C T$ manifestation in different timepoint after discharged. Note: The predominant pattern were ground-glass opacity (GGO), fibrous stripe. With time, the positive count of GGO, fibrous stripe and thickening of the adjacent pleura gradually decreased, while GGO and fibrous stripe showed obvious resolution during the first week and the third week after discharge, respectively

[54.4\%]), and thickening of the adjacent pleura (33 [22.1\%]) (Table 4). With time, the positive count of GGO, fibrous stripe and thickening of the adjacent pleura gradually decreased, while GGO and fibrous stripe showed obvious resolution during the first week and the third week after discharge, respectively (Fig. 1). Bronchovascular bundle distortion was found in 10 patients, with 4 instances of reversal (Fig. 2). GGO faded out with a temporary extension of area in 51 patients (Fig. 3).

\section{Discussion}

In this study, pulmonary lesions were found to be completely absorbed in $53.0 \%$ of patients during the 3rd week after discharge, implying that pulmonary damage caused by COVID-19 could be potentially repaired without any sequelae. However, more than $40 \%$ of patients demonstrated residual abnormalities, including GGO and fibrous stripe as the main CT manifestations at the 3-week radiological follow-up, for whom further radiological follow-up was continued. Two weeks was the appropriate median resolution period in this cohort.

The CT score of non-GGO lesions was used to evaluate residual pulmonary involvement. This was because an extended GGO area with decreased density may have occurred in some patients after discharge, and GGO is even a basic manifestation of convalescence, which could have led to over-estimation of the CT scores. As demonstrated in this study, patients with a CT score $\leq 1$ at discharge did not show a faster resolution than patients
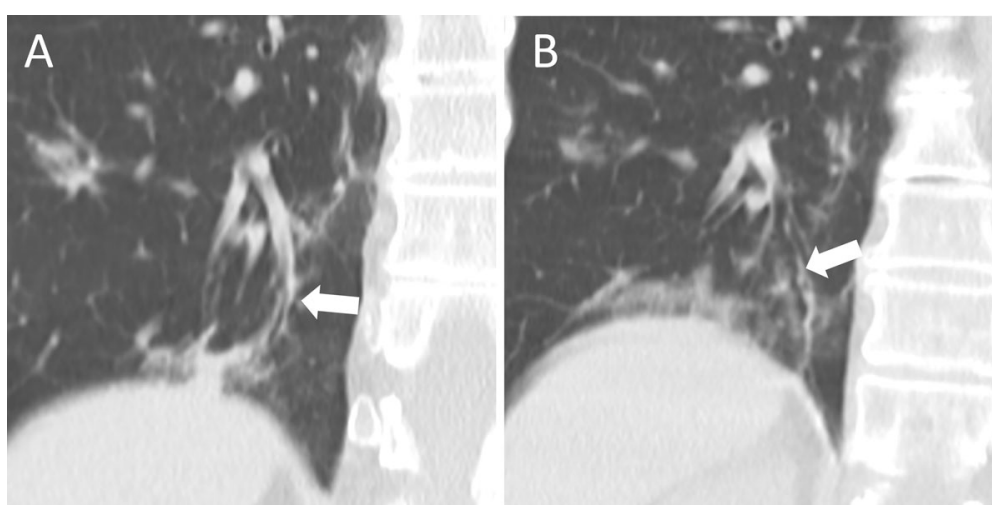

Fig. 2 Coronal chest CT images of bronchovascular bundle of COVID-19 pneumonia. Note: (a). Bronchovascular bundle distortion; (b). Bronchovascular bundle distortion reversed. All images have the same window level of -600 and window width of 1600 


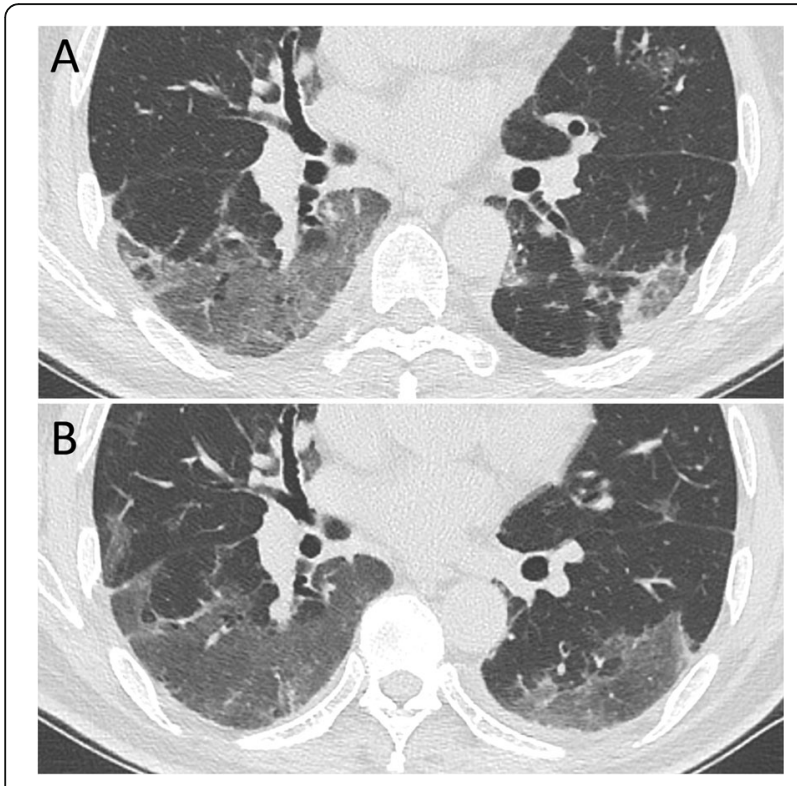

Fig. 3 "Tinted" sign. Note: (a). GGO in lower lobes of both lung; (b). GGO faded out with a temporary extension of area after 7 days. All images have the same window level of -600 and window width of 1600 with a CT score $>1$, which indicated a stable baseline in the CT scores of all patients at discharge.

Previous studies have reported that older age and male sex are high risk factors for worse outcomes in patients with COVID-19 [12, 13]. However, in this study, under the milieu of similar residual pulmonary lesions in the patients at discharge, females did not show a faster resolution rate. On the other hand, younger age was associated with a better outcome in the convalescence period in this study, which is consistent with a former study [14].

Radiological abnormalities have been reported to start resolving in the late phase in SARS, and the typical later-stage $\mathrm{CT}$ appearances were a coarse reticular pattern and GGO in the anterior part of the lungs [15]. For SARS, intralobular and interlobular septal thickening was observed to predominate over GGO even at 84 months [5]. In our cohort, GGO and fibrous stripe were the main imaging findings during the convalescence of COVID-19 pneumonia, which could be gradually absorbed completely, while a crazy-paving pattern was not demonstrated [3]. This observation addressed the question raised by Shi $\mathrm{H}$ et al. about whether the fibrosis in COVID-19 is irreversible [2]. There were three patterns in the residual lesions with proper evolution after discharge: 1. the extent of GGO was reduced and
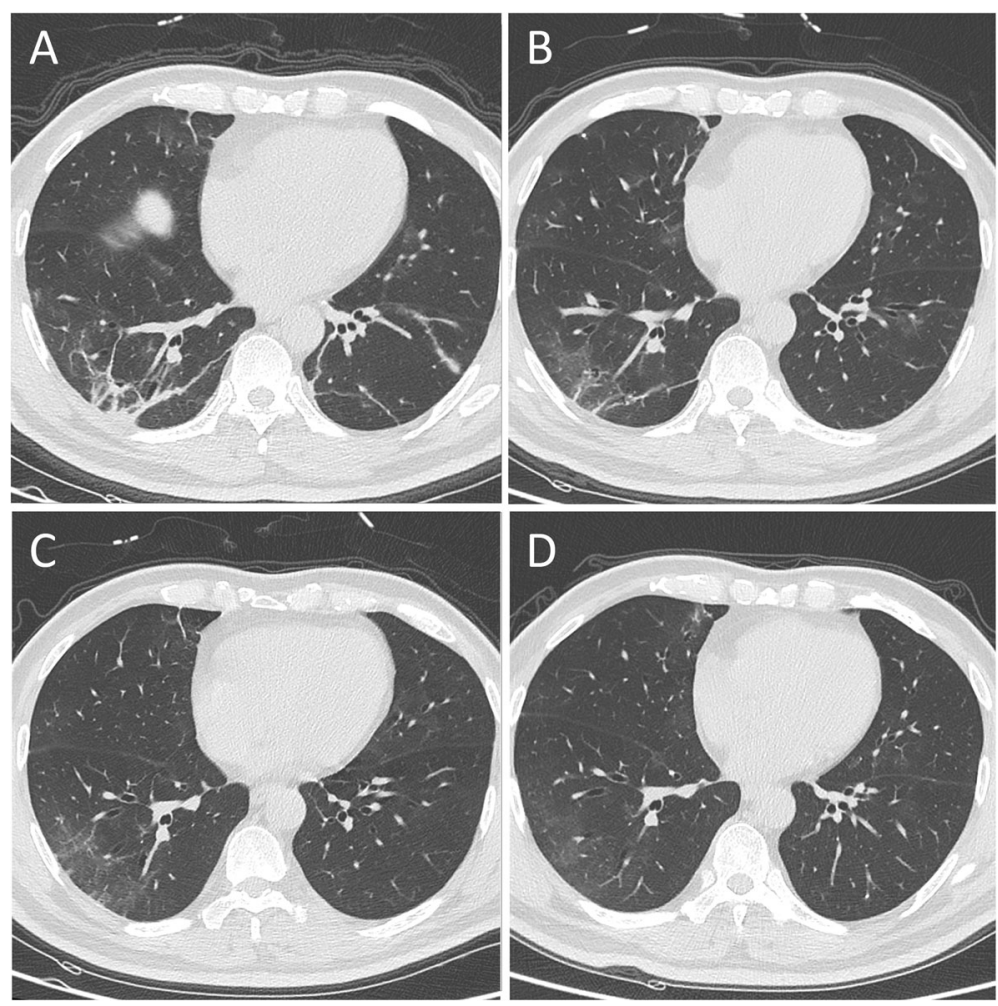

Fig. 4 Dynamic resolution procedure of fibrous stripes. Note: 73 year-old male (a). Fibrous stripes showed in lower lobes of both lung (b-d). Fibrous stripes gradually absorbed with time. All images have the same window level of -600 and window width of 1600 
gradually faded until it completely disappeared; 2 . fibrous stripes developed within the GGO area (mixed pattern), followed by gradual resorption and disappearance; and 3 . fibrous stripes gradually reduced with decreasing density (Fig. 4). "Tinted" sign and bronchovascular bundle distortion as two special features were discovered during the evolution. The "tinted" sign was demonstrated as extension of the GGO area and a decrease in density, which might follow the "melting sugar" sign [16]. In this study, 51 patients were found with a "tinted" sign. The appearance of this pattern probably implies the gradual resolution of inflammation with reexpansion of alveoli and hence the resolution or recovery of illness, for which the pathological evidence merits further investigation. In the mixed pattern, 10 patients were found to have bronchovascular bundle distortion, with 4 patients with complete resolution during 17-37 days after discharge. This may be caused by inflammatory distraction or subsegmental atelectasis. In addition, 14 patients had varying extents of residual fibrous stripes, and the lesions were not fully resorbed at the end of the observation, which may be attributed to the limited follow-up time. The other CT manifestations in convalescence, such as thickening of the adjacent pleura and small pleural effusion, could also be absorbed completely.

A main limitation of the current study is that no critical patients were involved in this study, as they were still hospitalized in our centre at the end of the study. Second, only semi-quantitative estimation was carried out in this study for the complicated radiological characteristics in the convalescent stage.

In summary, this study showed the dynamic resolution process of lung lesions in discharged patients recovering from COVID-19. For most of the discharged patients, two weeks after discharge might be the optimal time point for early radiological estimation. Elderly patients need a longer time to reach complete radiological resolution. This study may help to understand the recovery course of this disease and indicate an optimized time point for chest $\mathrm{CT}$ scans in discharged patients.

\section{Abbreviations}

COVID-19: Coronavirus Disease 2019; SARS-CoV: Severe acute respiratory syndrome coronavirus; CT: Computed Tomography; IQR: Interquartile ranges; GGO: ground-glass opacity; RT-PCR: Reverse-transcription-polymerase chainreaction

\section{Acknowledgements}

Not applicable.

\section{Authors' contributions}

$\mathrm{DL}$ and WZ designed the study and undertook most of the work, they should be regarded as co-first author. FP, LL, LY, DZ, JW and BL participated in data collection and analysis. All authors have contributed to the last version of the manuscript. The authors read and approved the final manuscript.
Funding

This study was not funded by anyone.

\section{Availability of data and materials}

The datasets used and/or analysed during the current study are available from the corresponding author on reasonable request.

\section{Ethics approval and consent to participate}

Ethics Committee of Union Hospital, Tongji Medical College, Huazhong

University of Science and Technology approved this study.

\section{Consent for publication}

Not applicable.

\section{Competing interests}

The authors declare that they have no competing interests.

\section{Author details}

'Department of Radiology, Union Hospital, Tongji Medical College, Huazhong University of Science and Technology, Jiefang Avenue No.1277, Wuhan 430022, China. ${ }^{2}$ Hubei Province Key Laboratory of Molecular Imaging, Wuhan 430022, China. ${ }^{3}$ MSC Clinical \& Technical Solutions, Philips Healthcare, Beijing 100000, China.

Received: 7 April 2020 Accepted: 4 May 2020

Published online: 24 May 2020

\section{References}

1. World Health Organization. Coronavirus disease 2019 (COVID-19) Situation report - 77 [https://www.who.int/docs/default-source/coronaviruse/ situation-reports/20200406-sitrep-77-covid-19.pdf?sfvrsn=21d1e632_2. Published on April 6, 2020].

2. Shi H, Han X, Jiang N, Cao Y, Alwalid O, Gu J, Fan Y, Zheng C. Radiological findings from 81 patients with COVID-19 pneumonia in Wuhan, China: a descriptive study. Lancet Infect Dis. 2020;20:425-34.

3. Pan F, Ye T, Sun P, Gui S, Liang B, Li L, Zheng D, Wang J, Hesketh RL, Yang $L$, Zheng C. Time Course of Lung Changes On Chest CT During Recovery From 2019 Novel Coronavirus (COVID-19) Pneumonia. Radiology. 2020: 200370.

4. Wang Y, Dong C, Hu Y, Li C, Ren Q, Zhang X, Shi H, Zhou M. Temporal changes of CT findings in 90 patients with COVID-19 pneumonia: a longitudinal study. Radiology. 2020:200843.

5. Wu X, Dong D, Ma D. Thin-section computed tomography manifestations during convalescence and long-term follow-up of patients with severe acute respiratory syndrome (SARS). Med Sci Monit. 2016;22:2793-9.

6. National Health Commission of the People's Republic of China. Diagnosis and treatment protocols of pneumonia caused by a novel coronavirus (trial version 7) [http://www.gov.cn/zhengce/zhengceku/2020-03/04/content_54 86705.htm. Published on March 3, 2020.].

7. Bruns AH, Oosterheert JJ, El Moussaoui R, Opmeer BC, Hoepelman Al, Prins $J M$. Pneumonia recovery: discrepancies in perspectives of the radiologist, physician and patient. J Gen Intern Med. 2010;25:203-6.

8. Chang YC, Yu CJ, Chang SC, Galvin JR, Liu HM, Hsiao CH, Kuo PH, Chen KY, Franks TJ, Huang KM, Yang PC. Pulmonary sequelae in convalescent patients after severe acute respiratory syndrome: evaluation with thinsection CT. Radiology. 2005;236:1067-75.

9. Franquet T. Imaging of pulmonary viral pneumonia. Radiology. 2011; 260:18-39.

10. Koo HJ, Lim S, Choe J, Choi SH, Sung H, Do KH. Radiographic and CT features of viral pneumonia. Radiographics. 2018;38:719-39.

11. Hansell DM, Bankier AA, MacMahon H, McLoud TC, Muller NL, Remy J. Fleischner society: glossary of terms for thoracic imaging. Radiology. 2008; 246:697-722.

12. Chen N, Zhou M, Dong X, Qu J, Gong F, Han Y, Qiu Y, Wang J, Liu Y, Wei Y, et al. Epidemiological and clinical characteristics of 99 cases of 2019 novel coronavirus pneumonia in Wuhan, China: a descriptive study. Lancet. 2020; 395:507-13.

13. Choi KW, Chau TN, Tsang O, Tso E, Chiu MC, Tong WL, Lee PO, Ng TK, Ng WF, Lee KC, et al. Outcomes and prognostic factors in 267 patients with severe acute respiratory syndrome in Hong Kong. Ann Intern Med. 2003; 139:715-23. 
14. Opal SM, Girard TD, Ely EW. The immunopathogenesis of sepsis in elderly patients. Clin Infect Dis. 2005;41(Suppl 7):S504-12.

15. Sheard S, Rao P, Devaraj A. Imaging of acute respiratory distress syndrome. Respir Care. 2012;57:607-12.

16. Pan Y, Guan H, Zhou S, Wang Y, Li Q, Zhu T, Hu Q, Xia L. Initial CT findings and temporal changes in patients with the novel coronavirus pneumonia (2019-nCoV): a study of 63 patients in Wuhan, China. Eur Radiol. 2020.

\section{Publisher's Note}

Springer Nature remains neutral with regard to jurisdictional claims in published maps and institutional affiliations.

Ready to submit your research? Choose BMC and benefit from:

- fast, convenient online submission

- thorough peer review by experienced researchers in your field

- rapid publication on acceptance

- support for research data, including large and complex data types

- gold Open Access which fosters wider collaboration and increased citations

- maximum visibility for your research: over $100 \mathrm{M}$ website views per year

At $\mathrm{BMC}$, research is always in progress.

Learn more biomedcentral.com/submissions 